\title{
FAST, ACCURATE MODELS FOR PREDICTING THE COMPLIANCE OF ELASTIC FLEXURE-JOINTED ROBOTS
}

\author{
Lael Odhner \\ Department of Mechanical Engineering, \\ Yale University \\ New Haven, Connecticut, USA \\ lael.odhner@yale.edu
}

\author{
Aaron Dollar \\ Department of Mechanical Engineering, \\ Yale University \\ New Haven, Connecticut, USA \\ aaron.dollar@yale.edu
}

\begin{abstract}
Robot manipulators having elastic links or flexure joints have a number of advantages, especially in simplifying the control of contact with other objects. However, current simplified parametric models of flexure motion do not accurately predict the behavior of these mechanisms under large deflections. This paper presents a "smooth curvature model" of flexure behavior that describes the curvature of a highly flexible member such as a flexure joint using a basis of three orthogonal polynomials. Using this model, we show that it is possible to predict the planar stiffness these mechanisms, even in cases where the deformation of the hinge is too large for the linear Euler-Bernoulli beam bending model. Using both finite element methods and the much less computationally expensive proposed model, numerical results will demonstrate that it is possible to accurately predict the in-plane compliance of a highly flexible mechanism in the presence of an external load. The results of this work are significant because they demonstrate that the behavior of flexure-based robotic mechanisms can be modeled quickly and with few parameters, enabling their use in closedloop control for situations where collision safety is a concern, and rigorous model-based path planning for obstacle avoidance, among other applications.
\end{abstract}

\section{INTRODUCTION}

For many years, there has been a general recognition that physical or simulated compliance can be used to improve the ability of a robot to perform certain tasks, specifically tasks involving contact with the environment, such as grasping, manipulation, and locomotion [1-5]. Mechanical compliance in a robot hand, such as the SDM Hand shown in Fig. 1, simplifies control of a robot because contact does not need to be exactly predicted or modeled. The robot can simply be commanded to collide with an object, and the soft contact will ensure that the load is limited by compliance, or balanced between multiple contacts through elastic averaging. Another key advantage of
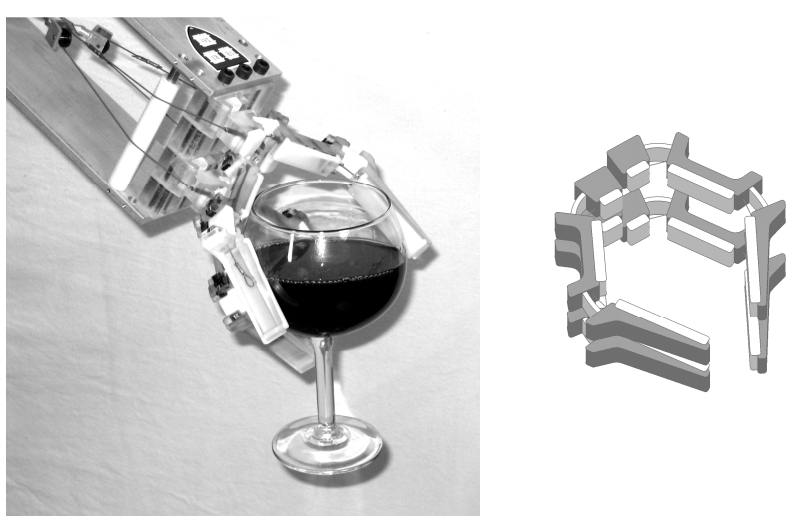

Figure 1. Left: the SDM Hand, a flexure-based, underactuated robotic hand designed by the authors. Right: a computational model of the SDM Hand created to study compliant finger behavior

planned-in compliance is robustness to unforeseen collisions with objects in an uncertain environment [6].

One obstacle to progress in this field is the lack of good constitutive models for large-deformation flexure joints. These elastic elements are often modeled as Euler-Bernoulli beams connecting two rigid bodies. Because the beam bending equations are nonlinear for large deformation, approximated beam profiles are often used in place of exact solutions [7]. For example, a flexure is sometimes modeled as an arc of constant curvature [8], or as a single rotational degree of freedom at the center of the joint [9]. These methods are capable of estimating the gross kinematic behavior, in the sense that the angles between the manipulator's rigid bodies are described; as a consequence, these models are useful for inverse kinematics or motion planning. However, the obvious drawback to these single-parameter models is their inability to predict the compliance of a flexure. A one-degree of freedom model will, by definition, admit motion in one direction locally, and it will be infinitely stiff in any other direction. This problem is shared by other, more accurate models such as the family of pseudorigid body models [10]. The remaining alternatives are finite 
element methods [11], or modal methods [12-13]. Rather than having too few degrees of freedom, finite element methods describe the bending of a continuum beam with many degrees of freedom, rendering real-time, model-based control or planning very difficult. The modal approach, however, represents the motion of the flexure as a linear combination of limited number of shape functions that serve as a basis for describing the shape of the flexure.

This paper introduces a simple, modal model for planar flexure motion, based on the assumption that the curvature of the flexure is a smooth function, and can be approximated by a low-order basis of orthogonal polynomials. This elegant model describes the shape of a flexure hinge accurately enough that the Jacobian and Hessian of the kinematics can be used to predict the generalized force and stiffness of a robotic mechanism. Consequently, it can be used as a general-purpose, rather than special-purpose, model for planar flexure joints.

We will proceed in the following order: First, the smooth curvature model will be introduced, and the method of taking derivatives to find force balance and stiffness will be shown. The accuracy of the model will be briefly demonstrated by comparing the exact solution of Bernoulli's rectangular elastica problem to the solution obtained by the smooth curvature model. Then, we will demonstrate how this model can be used to accurately predict the in-plane stiffness of a flexure-based serial link chain to forces at multiple points along the manipulator. Lastly, the results of the smooth curvature model will be cross-checked by finite element beam models approximating the same model and the same loading conditions.

\section{NOMENCLATURE}

$s$
$\kappa(s)$
$\varphi(s)$
$x(s)$
$y(s)$
$\tau(s)$
$L$
$E, I$
$x_{\text {tip }}, y_{\text {tip }}, \varphi_{\text {tip }}$
$q=\left[q_{0}, q_{1}, q_{2}\right]^{\mathrm{T}}$
$f_{x}, f_{y}, f_{\varphi}$
$U$
$\mathbf{J}_{p}$
$\mathbf{H}_{p}$
$\mathrm{~K}$
$\mathbf{C}_{p}$

Arc length along the flexure

Flexure curvature profile

Flexure angular profile

Flexure $x$ profile

Flexure $y$ profile

The torque profile along the flexure.

Flexure length

Flexure elastic modulus \& bending moment

Flexure tip position and orientation

Generalized flexure coordinates

The force and moment on the flexure tip

The elastic energy of the flexure

The Jacobian of some point on a robot, $\underline{p}$

The Hessian of some point on a robot, $p$

The generalized stiffness matrix of a robot

The compliance of a robot at some point, $\underline{p}$

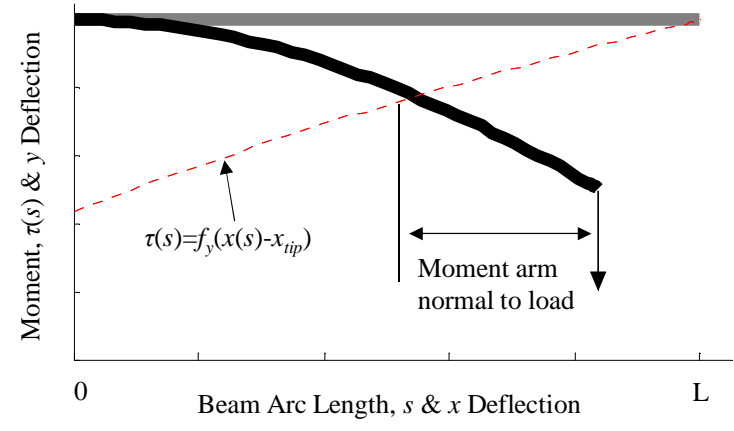

Figure 2. The moment profile of a beam undergoing large deformation due to a vertical load.

\section{A BETTER MODAL BEAM MODEL}

\section{Bernoulli’s Beam Bending Model}

Bernoulli's model for flexible beams posits that the elastic deformation of a beam in bending can be modeled as a continuous rotation of the beam about some neutral axis. The curvature of the beam, $\kappa(s)$, is the derivative of the beam angle $\varphi(s)$ as a function of the arc length, $s$,

$\frac{d \varphi}{d s}=\kappa(s)=\frac{\tau(s)}{E I}$

Here $\tau(s)$ is the torque exerted on the beam at any point. The $x$ and $y$ profile of the beam can be found by integrating the angular profile,

$\frac{d x}{d s}=\cos (\varphi(s))$

$\frac{d y}{d s}=\sin (\varphi(s))$

These equations, taken together as a system, are difficult to solve because the torque on a beam loaded at the end is a function of $x(s)$ and $y(s)$.

$$
\frac{d}{d s}\left[\begin{array}{c}
\kappa \\
\varphi \\
x \\
y
\end{array}\right]=\left[\begin{array}{c}
\left(f_{x} \sin (\varphi)-f_{y} \cos (\varphi)+f_{\varphi}\right) / E I \\
\kappa \\
\cos (\varphi) \\
\sin (\varphi)
\end{array}\right]
$$

The torque profile can be thought of as a tip moment $f_{\varphi}$ plus an end load, multiplied by the projected distance normal to the direction of applied tip load $\left(f_{x}, f_{y}\right)$, as shown in Fig. 2 for the simple case of a beam undergoing a purely vertical load. To 
describe the beam profile without solving this equation, we can make a few simplifying assumptions. First, as Fig. 2 illustrates, the variation of the torque with the length of the beam is fairly smooth, because the profile of the beam is smooth. Thus, it would be reasonable to approximate the torque, and hence the curvature, as having a linear or quadratic profile as a function of beam length. This model will be reasonable as long as the flexure force and curvature of the flexure are small enough that the bending profile is smooth.

\section{Model Order Choice}

The model presented in this paper approximates the curvature of a bending beam as a three-parameter polynomial function of arc length. The primary reason for this choice was the insight that at minimum, three parameters are needed to independently describe the behavior of a flexure joint: the tip position $\left(x_{t i p}, y_{t i p}\right)$ and orientation, $\varphi_{t i p}$. These three values can be used to describe a flexure joint in Denavit-Hartenberg notation, as a transformation from one link to the next. It makes sense, then, to include at least three parameters in the flexure model. It also makes sense to avoid the inclusion of any more parameters, because a larger number of parameters would guarantee that there will be some internal degrees of freedom in the flexure that do not result in any motion of the rigid links to which it is attached. As a result, the equations of motion would have an extremely ill-conditioned mass matrix as a result of having to simulate internal vibrational modes of the flexure.

The quadratic model of flexure curvature was written in a basis of orthogonal polynomials, namely Legendre polynomials that have been translated and scaled to be orthogonal over the length of the flexure, from $s=0$ to $s=L$,

$\kappa(s, \underline{q})=q_{0} \frac{1}{L}+q_{1} \frac{2 s-L}{L^{2}}+q_{2} \frac{6 s^{2}-6 s L+L^{2}}{L^{3}}$

The parameters $q_{0}, q_{1}$, and $q_{2}$ act as a generalized coordinate vector describing the shape and the elastic energy of the flexure.

\section{Flexure Shape}

Because a flexure joint serves to connect two rigid links together, the critical aspect of the flexure shape that must be captured is the location of the flexure endpoint, $\left(x_{t i p}, y_{t i p}, \varphi_{t i p}\right)$. The angular profile, $\varphi(s, q)$, is the integral of the curvature,

$$
\begin{aligned}
\varphi(s, \underline{q}) & =\int \kappa(s, \underline{q}) d s \\
& =q_{0} \frac{s}{L}+q_{1} \frac{s^{2}-s L}{L^{2}}+q_{2} \frac{2 s^{3}-3 s^{2} L+s L^{2}}{L^{3}}
\end{aligned}
$$

At the end of the flexure ( $s=L$ ), the tip angle is equal to $q_{0}$. This is a happy side effect of using orthogonal polynomials, as all the higher, non-constant terms must integrate to zero:

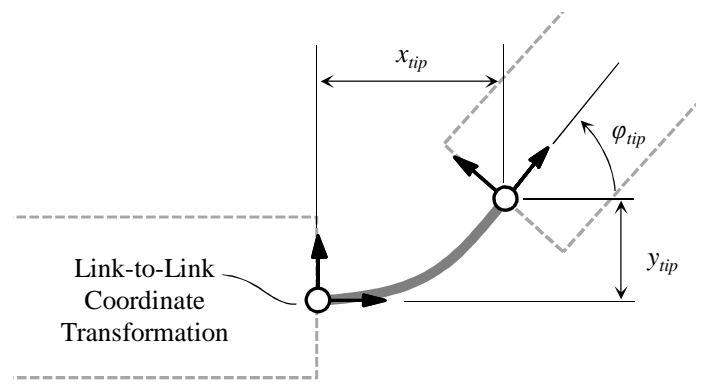

Figure 3 . The relative position and orientation of each link in a flexure-based robot can be described by three parameters, $x_{t i p}, y_{t i p}$ and $\varphi_{t i p}$.

$\varphi_{\text {tip }}=\varphi(L, \underline{q})=q_{0}$

The tip position of the flexure can be found by integrating the cosine and sine of the angular profile,

$$
\begin{aligned}
& x_{t i p}=\int_{0}^{L} \cos (\varphi(s, \underline{q})) d s \\
& y_{t i p}=\int_{0}^{L} \sin (\varphi(s, \underline{q})) d s
\end{aligned}
$$

These expressions are transcendental, so direct calculation is very difficult. Rather than trying to use the transcendental solution, it is better to use a polynomial interpolation for cosine and sine, so that (8) and (9) can be analytically integrated. If the range of flexure bending is limited to $90^{\circ}$ or less, the interpolating function only has to be accurate on a limited interval. Any good interpolating method (such as Chebyshev interpolation [14]) will produce a low order polynomial that can be used to calculate $x_{\text {tip }}$ and $y_{\text {tip }}$ accurately.

\section{Elastic Energy}

A Lagrangian model of a machine element can be described by generalized coordinate using a shape function, which has just been computed, and a function describing the energy of the system in any configuration. In this case, Bernoulli's model provides a constitutive description of the energy in a bent flexure:

$U(\underline{q})=\frac{E I}{2} \int_{0}^{L} \kappa(s, \underline{q})^{2} d s$

If the parameterized curvature from (5) is substituted in here, the resulting energy function is a weighted sum of squared parameters,

$U(\underline{q})=\frac{E I}{2 L}\left(q_{0}^{2}+\frac{q_{1}^{2}}{3}+\frac{q_{2}^{2}}{5}\right)$ 
Because the polynomial basis used to represent the curvature is orthogonal, there are no cross-terms in this expression.

\section{Summary}

So far, a model has been developed, based on the assumption that the curvature of a bending beam can be approximated using low-order, orthogonal polynomials. This modal assumption can be carried forward to find the position and orientation of the beam tip as a function of the modal coordinates, and also the energy of the beam in any bent configuration describable by the coordinates. In the following sections, we will demonstrate how this model can be applied to accurately describe the deformation and stiffness of flexure hinges.

\section{FORCE AND EQUILIBRIUM}

\section{The Principle of Virtual Work}

The advantage of describing the deformation of a flexure in generalized coordinates rather than as a differential equation is the applicability of standard tools for robot analysis. For example, suppose that a manipulator with flexure joints is loaded with a force $f_{p}$ at a point $\underline{p}$. The balance of generalized forces, $\underline{F}$, for any configuration $q$ can be described by Castigliano's theorem looking at the derivative of the energy function with respect to $q$ :

$$
\underline{F}=\nabla_{q} U(\underline{q})+\mathbf{J}_{p}(\underline{q})^{T} \underline{f}
$$

Here $\mathbf{J}_{p}(q)$ is the Jacobian of the point $\underline{p}$. When the right hand side of this expression is equal to zero, then $q$ is an equilibrium configuration. For a mechanism having multiple flexure hinges, each hinge will be kinematically described by a transformation matrix,

$$
T=\left[\begin{array}{ccc}
\cos \left(\varphi_{t i p}\right) & -\sin \left(\varphi_{t i p}\right) & x_{t i p} \\
\sin \left(\varphi_{t i p}\right) & \cos \left(\varphi_{t i p}\right) & y_{t i p} \\
0 & 0 & 1
\end{array}\right]
$$

The Jacobian of any point on the mechanism can be computed by the product rule, and will involve the partial derivatives of each transformation with respect to its joint variables. For the purpose of modeling a serial manipulator having flexure joints, the partial derivatives of (7), (8) and (9) can be computed,

$$
\begin{aligned}
& \frac{\partial \varphi_{t i p}}{\partial q_{i}}= \begin{cases}1, & i=0 \\
0, & i \neq 0\end{cases} \\
& \frac{\partial x_{t i p}}{\partial q_{i}}=-\int_{0}^{L} \sin (\varphi(s, \underline{q})) \frac{\partial \varphi_{t i p}}{\partial q_{i}} d s
\end{aligned}
$$

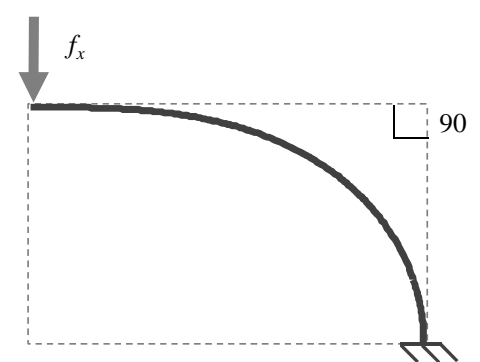

Figure 4. Bernoulli's rectangular elastica is the curve formed by bending a cantilevered beam to a $90^{\circ}$ angle using a compressive force $f_{x}$.

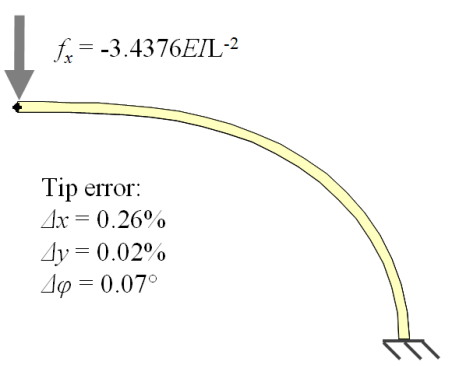

Figure 5. The smooth curvature model reproducing the shape of the rectangular elastica.

$\frac{\partial y_{t i p}}{\partial q_{i}}=\int_{0}^{L} \cos (\varphi(s, \underline{q})) \frac{\partial \varphi_{t i p}}{\partial q_{i}} d s$

The trigonometric functions in (15) and (16) can be integrated by applying a Chebyshev interpolation, as was previously done. Alternatively, the polynomial expressions resulting from the interpolation and integration of (8) and (9) can be directly differentiated. The gradient of the energy function from (11) can be written as a matrix multiplication,

$\nabla_{q} U(\underline{q})=\frac{E I}{L}\left[\begin{array}{ccc}1 & 0 & 0 \\ 0 & 1 / 3 & 0 \\ 0 & 0 & 1 / 5\end{array}\right]\left[\begin{array}{l}q_{0} \\ q_{1} \\ q_{2}\end{array}\right]$

\section{Finding Equilibrium under Load}

To demonstrate that this model accurately captures the nonlinear deformation of a flexure in bending, the modal solution to a canonical problem in nonlinear deformation is presented here. The rectangular elastica problem, posed by Jacob Bernoulli in 1692 [15], is the problem of finding the compressive load which, when applied to the tip of a beam, causes it to bend over to $90^{\circ}$, as depicted in Fig. 4. The exact solution to this problem is well-known, and it can be computed numerically by straightforward integration of (4) as a two-point boundary value problem. The method of solution is to fix the 


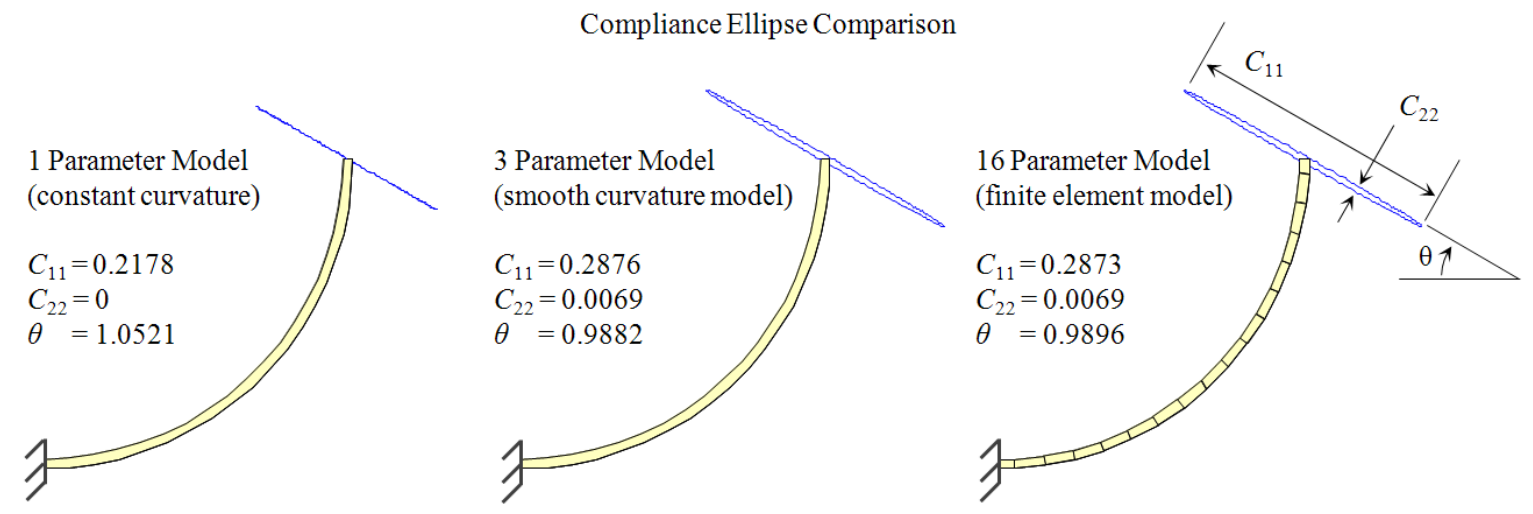

Figure 6. The tip compliance ellipses of the constant curvature model, the smooth curvature model, and a 16 element finite element flexure model.

angle at the tip of the beam to $90^{\circ}$, iteratively integrating and choosing the value of compressive load yielding a $0^{\circ}$ angle at the base of the beam. The value of the compressive force $f_{x}$ resulting in this condition can be found as a function of $E, I$ and $L$

$$
\frac{f_{x} L^{2}}{E I}=-3.4376 \ldots
$$

When this force is applied to the end of the beam in the form of (12), the equilibrium position of the smooth curvature model matches the exact solution within $0.26 \%$ in $x, 0.02 \%$ in $y$, and $0.07^{\circ}$ in $\varphi$. Figure 5 plots the deformed profile of the smooth curvature beam, with an asterisk indicating the true position of the beam tip for the rectangular elastica curve.

\section{STIFFNESS}

The previous section briefly demonstrated how the smooth curvature model can be used to find the equilibrium shape of a beam under load, even for large angle deformation. The more critical question is the ability of this model to predict the compliance of a manipulator. By looking at the local variation in net generalized force as a function of $q$, the generalized stiffness $\mathbf{K}(q)$ can be written:

$\mathbf{K}(\underline{q})=\nabla_{q} \nabla_{q} U(\underline{q})+\mathbf{H}_{p}(\underline{q})^{T} \underline{f}_{p}$

The Hessian of point $\underline{p}, \mathbf{H}_{p}(q)$, is the tensor of partial derivatives of $\mathbf{J}_{p}(q)$. As before with the Jacobian, the Hessian of any point is calculated using the partial derivatives of each joint transformation matrix. The derivatives (14), (15) and (16) can be again taken:

$$
\frac{\partial^{2} \varphi_{t i p}}{\partial q_{i} \partial q_{J}}=0
$$

$$
\begin{aligned}
& \frac{\partial^{2} x_{t i p}}{\partial q_{i} \partial q_{J}}=- \int_{0}^{L} \sin (\varphi(s, \underline{q})) \frac{\partial^{2} \varphi_{t i p}}{\partial q_{i} \partial q_{J}} d s- \\
& \int_{0}^{L} \cos (\varphi(s, \underline{q})) \frac{\partial \varphi_{t i p}}{\partial q_{i}} \frac{\partial \varphi_{t i p}}{\partial q_{J}} d s \\
& \frac{\partial^{2} y_{t i p}}{\partial q_{i} \partial q_{j}}=\int_{0}^{L} \cos (\varphi(s, \underline{q})) \frac{\partial^{2} \varphi_{t i p}}{\partial q_{i} \partial q_{j}} d s- \\
& \int_{0}^{L} \sin (\varphi(s, \underline{q})) \frac{\partial \varphi_{t i p}}{\partial q_{i}} \frac{\partial \varphi_{t i p}}{\partial q_{j}} d s
\end{aligned}
$$

As before, the result of this is a polynomial expression for each partial derivative. The energy Hessian is easily derived from (17),

$$
\nabla_{q} \nabla_{q} U(\underline{q})=\frac{E I}{L}\left[\begin{array}{ccc}
1 & 0 & 0 \\
0 & 1 / 3 & 0 \\
0 & 0 & 1 / 5
\end{array}\right]
$$

\section{Finding the Compliance of a Point on a Robot}

Once the generalized stiffness matrix for a system, $\mathbf{K}(q)$, has been found, the compliance $\mathbf{C}_{p}$ of a point $\underline{p}$ on the robot can be found using the Jacobian of that point's coordinates [16],

$$
\mathbf{C}_{p}(\underline{q})=\mathbf{J}_{p}(\underline{q}) \mathbf{K}(\underline{q})^{-1} \mathbf{J}_{p}^{T}(\underline{q})
$$

This could be used, for example, to describe the compliance "ellipses" along the surface of a gripper, so that a controller had some confidence about how it could move without being damaged in an accidental collision. As a simple illustration of how well the smooth curvature model predicts stiffness, a flexure was modeled using a constant curvature model [8], the proposed three parameter model, and with a 16 element finite element Euler-Bernoulli model, vis. [11]. The constant curvature model is essentially the truncation of the smooth 


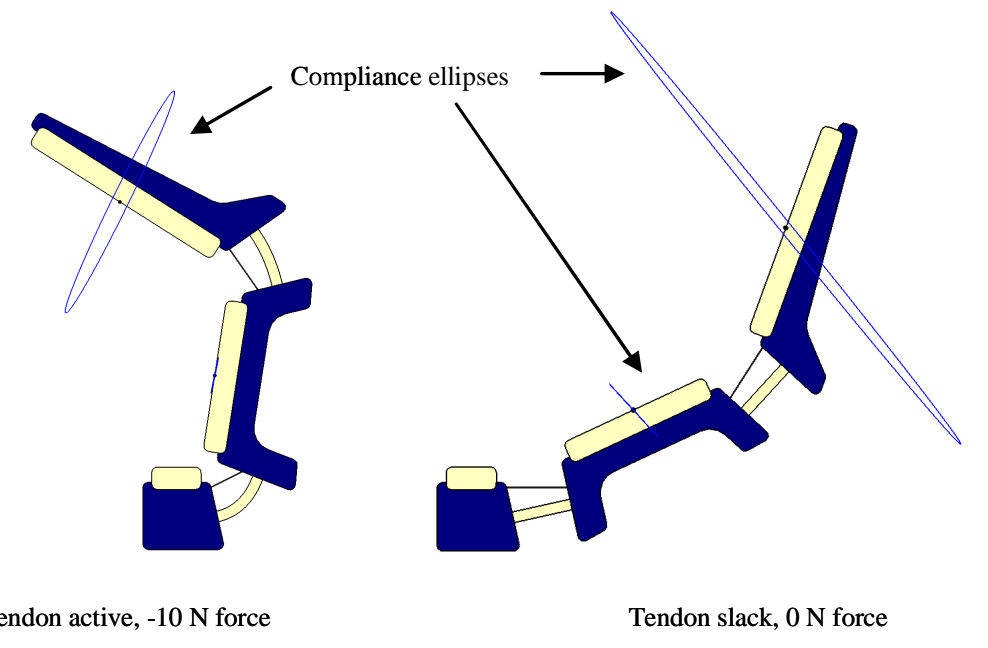

Figure 7. Compliance ellipses for the two-link SDM fingers.

curvature model to one parameter. A complete description of the constant curvature model kinematics can be found in [8]. The beam parameters were non-dimensionalized, so that $\mathrm{E}=\mathrm{I}=\mathrm{L}=$ 1. A pure moment was applied to the tip of each beam, bending it to $90^{\circ}$. This served as a fair comparison case because the solution to this loading condition is an arc, a shape that the constant curvature model is capable of reproducing.

As the results in Fig. 6 demonstrate, the smooth curvature model agrees to with the finite element model to within $0.15 \%$. The constant curvature model under-predicts the compliance of the flexure tip by $24 \%$ in the direction of maximum compliance. In the direction of minimum compliance, the constant curvature model fails to predict any compliance, which is expected due to the fact that there are fewer degrees of freedom in the model than there are directions in the plane.

\section{Two-Link Finger Models}

To demonstrate that the smooth curvature model can be used to predict compliance of larger structures, a model of a two-link finger from the authors' SDM Hand [6] was modeled in Denavit-Hartenberg form in Matlab. The finger consists of two links connected by flexures, driven by a single tendon that runs through the middle link to the distal link, where it is anchored. Figure 7 depicts this hand in two configurations: at the left, the tendon is pulled with a force that is large enough to close the hand. At the right, the tendon is slack, so the finger hangs limp. The compliance of the center of each pad on the finger was predicted using (24), and the resulting compliance ellipses are overlaid onto the images. The result of this analysis was compared to a finite element model of the same finger, using 16 rotational elements to model each flexure joint. Table 1 compares the results from the finite element model to the results from the smooth curvature model. The results indicate that these two modeling techniques agree closely for predicting the stiffness of the finger. This is encouraging, because the vast

\begin{tabular}{|l|l|l|l|}
\hline \multicolumn{3}{|c|}{ Table 1:Finger Compliance, FEM vs. Smooth Curvature } \\
\hline Case & Parameter & FEM & $\begin{array}{l}\text { Smooth } \\
\text { Curvature }\end{array}$ \\
\hline \multirow{2}{*}{$\begin{array}{l}\text { Tendon } \\
\text { active, } \\
\text { middle pad }\end{array}$} & Max Compliance & $1.031 \times 10^{-2}$ & $1.032 \times 10^{-2}$ \\
\cline { 2 - 4 } & Min Compliance & $1.926 \times 10^{-4}$ & $1.927 \times 10^{-4}$ \\
\cline { 2 - 4 } & Maj. Axis Angle & $78.251^{\circ}$ & $78.350^{\circ}$ \\
\hline \multirow{2}{*}{$\begin{array}{l}\text { Tendon } \\
\text { active, distal } \\
\text { pad }\end{array}$} & Max Compliance & $6.992 \times 10^{-2}$ & $7.000 \times 10^{-2}$ \\
\cline { 2 - 4 } & Min Compliance & $4.627 \times 10^{-3}$ & $4.625 \times 10^{-3}$ \\
\cline { 2 - 4 } & Maj. Axis Angle & $63.865^{\circ}$ & $63.968^{\circ}$ \\
\hline \multirow{2}{*}{$\begin{array}{l}\text { Tendon } \\
\text { slack, } \\
\text { middle pad }\end{array}$} & Max Compliance & $1.970 \times 10^{-2}$ & $1.970 \times 10^{-2}$ \\
\cline { 2 - 4 } & Min Compliance & $1.017 \times 10^{-4}$ & $1.022 \times 10^{-4}$ \\
\cline { 2 - 4 } & Maj. Axis Angle & $-47.399^{\circ}$ & $-47.397^{\circ}$ \\
\hline \multirow{2}{*}{$\begin{array}{l}\text { Tendon } \\
\text { slack, distal } \\
\text { pad }\end{array}$} & Max Compliance & $1.546 \times 10^{-1}$ & $1.546 \times 10^{-1}$ \\
\cline { 2 - 4 } & Min Compliance & $3.146 \times 10^{-3}$ & $3.146 \times 10^{-3}$ \\
\cline { 2 - 4 } & Maj. Axis Angle & $-50.971^{\circ}$ & $-50.971^{\circ}$ \\
\hline
\end{tabular}

reduction in the number of parameters needed to describe the finger will enable faster, more compact models that can hopefully be used to make real-time decisions about path planning, grasping and manipulation.

\section{CONCLUSIONS}

This paper has introduced a new model for planar flexure joints that consistently represents the flexure's first-order and second-order behavior, for example, the balance of forces as represented by the Jacobian and the stiffness as represented by the Hessian. This is extremely important if the analysis of flexure-based robotic mechanisms such as the SDM hand is to be formalized to a greater degree. There is not space in this paper to exhaustively explore the accuracy of the model; the authors are working on a longer paper that examines the accuracy of this model in predicting both the deformation of the 
flexure under arbitrary end loads, as well as the ability of the smooth curvature model to predict the first several buckling modes of an end-loaded flexure. Buckling is an important phenomenon to model accurately, especially in grasping and manipulation, where a buckling mode could represent a way in which the object can pop or twist out of a gripper.

Another appealing direction of future research is the examination of "massless" flexure modeling, that is, studying how the inertial matrix of a manipulator scales as the flexures become lighter and lighter. It seems possible that the small number of parameters used by the smooth curvature model could render this model quite useful for dynamic simulation and inverse dynamics.

As always, a few caveats are in order with this model. First, all of the beam models used here are Euler-Bernoulli beam models, so they disregard deformation in tension and shear. Users would do well to determine whether their flexure can be reasonably modeled using the Euler-Bernoulli model, or, if not, whether correction factors exist for coarse approximation. The biggest thing to keep in mind, however, is that most flexure hinges also have a great deal of out-of-plane compliance. Just as the constant curvature model under-predicts the in-plane compliance of a flexure, the smooth curvature model predicts no out-of-plane compliance whatsoever. Better modal models of out-of-plane flexure behavior are an appealing direction of future research.

\section{REFERENCES}

[1] H. Hanafusa and H. Asada, "Stable Prehension by a Robot Hand with Elastic Fingers," Proceedings of the 7th International Symposium on Industrial Robots, pp. 361-368, 1977.

[2] S. Sur and R.M. Murray, "An Experimental Comparison of Tradeoffs in Using Compliant Manipulators for Robotic Grasping Tasks," Proceedings of the 1997 IEEE International Conference on Robotics and Automation, pp. 1807-1814, 1997.

[3] M. Cutkosky and I. Kao, "Computing and controlling compliance of a robotic hand," IEEE Transactions on Robotics and Automation, v.5, n.2, pp. 151-165, 1989.

[4] G. Pratt, "Low impedance walking robots," J. Integrative and Comparative Biology, v.42, n.1, p.174, 2002.

[5] S. Kim, M. Spenko, S. Trujillo, B. Heyneman, D. Santos, and M. Cutkosky, "Smooth vertical surface climbing with directional adhesion," IEEE Transactions on Robotics, v.24, n.1, pp.65-74, 2008 .

[6] A. Dollar and R. Howe, "A robust compliant grasper via shape deposition manufacturing," IEEE/ASME Transactions on Mechatronics, vol. 11(2), pp.154-161, 2006.
[7] N. Lobontiu, "Distributed-Parameter dynamic model and optimized design of a four-link pendulum with flexure hinges," Mechanism and Machine Theory, v. 36, pp. 653-669, 2001.

[8] M. Hannan and I. Walker, "Kinematics and the implementation of an elephant's trunk manipulator and other continuum style robots," Journal of Robotics Systems, v. 20, n. 2, pp. 45-63, 2003.

[9] M. Ciocarlie and P. Allen, "A Design and Analysis Tool for Underactuated Compliant Hands," Proceedings of the 2009 IEEE/RSJ International Conference on Intelligent Robotics and Systems, pp. 5235-5239, 2009.

[10] A. Midha, T. Norton, and L. Howell, "On the nomenclature, classification, and abstractions of compliant mechanisms," Trans. of the ASME, v. 116, n. 1, pp.270-280, 1994.

[11] E. Bayo, "A finite-element approach to control the endpoint motion of a single-link flexible robot," Journal of Robotic Systems, vol. 4(1), pp. 63-75, 1986.

[12] S. Timoshenko and D. Young, Advanced Dynamics, p. 204, McGraw-Hill, 1948.

[13] G. Chirikjian and J. Burdick, "A modal approach to hyperredundant manipulator kinematics," IEEE Trans. on Robotics and Automation, v. 10, n. 3, pp. 343-354, 1994.

[14] W. Press, S. Teukolsky, W. Vetterling, and B. Flannery, Numerical Recipes in $C, 2^{\text {nd }}$ ed., p. 190, Cambridge University Press, 1992.

[15] R. Levien, "The elastica: a mathematical history," Technical Report No. UCB/EECS-2008-103, University of California at Berkeley, 2008.

[16] H. Asada, J.J.E. Slotine, Robot Analysis and Control, p. 88, John Wiley \& Sons, 1986. 